\title{
Management of Phyllodes Tumour of the Breast at Ain Shams University Hospital, Egypt: A Retrospective Review of a Rare Disease
}

\author{
Mohamed G Qassem MD, MRCS; Karim Fahmy, MD; MRCS; Rania Elahmady, MD \\ Department of General Surgery, Ain Shams University, Egypt
}

Background: Phyllodes tumors are rare distinctive fibro-epithelial tumors of the breast and their management continues to be questioned. Between $10 \%$ and $40 \%$ of phyllodes tumors have a tendency for LR and general dissemination. The aim of our study was to review the management of Phyllodes Tumour (PT) and to examine the determinants of local recurrence (LR).

Methods: A retrospective cohort study of female patients with histologically proven PT who presented to Ain Shams University Hospital (ASUH) between August 2014 and August 2018. Data collected from their files were analyzed to correlate LR after surgical intervention to demographic characteristics, tumour site, tumour size, tumour pathological grade, safety margin and type of performed surgery.

Results: Our study included 24 patients who presented to ASUH and were diagnosed to have PT, with follow up a mean of 30 months \pm 10 . Only 6 patients (25\%) demonstrated LR after the surgical intervention by a mean of 1.250 years \pm 0.354 (range 1-1.5 years). In the recurrent group, the mean age was 39.333 (range 27-51) (P 0.636), the lesion size was between 5-15 cm (mean 11.667) (P 0.016), and safety margin was exceeding $10 \mathrm{~mm}$ ( $\mathrm{P} 0.422$ ).

Conclusion: Benign and small PTs should be removed with safety margin rather than simple excision, and their malignant potential should be considered. Wider-scale studies are vital in understanding the recurrence behavior of this rare disease.

Key words: Phyllodes tumour, local recurrence, safety margin.

\section{Introduction}

Phyllodes tumor is a rare tumor of the breast in comparison to other histologic subtypes, however, itself, is not a rare tumor, accounting for $<1 \%$ of all breast malignancies, ${ }^{1}$ and has an incidence of about 2.1 per million. Most of these tumors are benign, but some have a malignant potential. These tumors commonly occur in females during the $4^{\text {th }}$ or 5th decade of life. Phyllodes tumors usually present as mobile painless breast masses, however, approximately $20 \%$ of tumors are identified on screening mammography and are non-palpable. ${ }^{2}$

Although phyllodes tumors are similar to fibroadenomas, suspicion for a phyllodes tumor is based on large size, a rapid growth rate, and findings of stromal hyperplasia and atypia on microscopic examination. ${ }^{2}$ The stromal cellularity, atypia and mitotic index are the main parameters in the differentiation of phyllodes tumors from fibroadenomas and in distinguishing a benign from a malignant phyllodes tumor. ${ }^{3}$ The World Health Organization (WHO) categorizes phyllodes tumors According to the degree of stromal hyperplasia and atypia, as benign, borderline and malignant with malignant tumors accounting for $25 \%$ of resected tumors. ${ }^{4}$

Till now, Surgery is the main line of treatment.
National Comprehensive Cancer Network (NCCN) guidelines for the management of phyllodes tumors recommend wide excision with margins $\geq 1 \mathrm{~cm}$ and recommend no axillary intervention. ${ }^{5}$

Main determinants of local recurrence in phyllodes tumors include mitotic activity, tumor margin, and stromal cellular atypia. The local recurrence rates following wide local excision are $8 \%$ for benign phyllodes tumors and $21-36 \%$ for borderline and malignant tumours. ${ }^{6}$ The objective of this retrospective study is to review the management of the rarely-occurring Phyllodes Tumour at our institution with special highlighting of the potential determinants of its local recurrence.

\section{Patients and methods \\ Study eligibility criteria:}

Following are characteristics of individual studies required for their inclusion in this study depicted into the PICOS format:

- Participants: All female adult patients with histologically proven Phyllodes Tumour (PT). Those patients presented to Breast Outpatient Clinic of Ain Shams University Hospital (ASUH) in the period between August 2014 and August 2018. They had triple assessment and routine pre-operative investigations (Figure 1). Management plans were based 
upon the discussions in weekly-held Multidisciplinary meetings (MDM). Follow up was arranged to be done bi-annually.

- Intervention (Exposure): Mastectomy, Wide Local Excision (WLE) or Oncoplastic Surgery (OS) (Figure 2).

- Control: Patients who did not show recurrence after the planned surgical intervention.

- Outcomes: Local recurrence (LR) after surgical intervention, with correlation to demographic characteristics, tumour site, tumour size, tumour pathological grade, safety margin and type of performed surgery.

- Study design: Retrospective cohort study of prospectively collected data.

All patients were well informed and signed informed consent prior to surgery. This study was approved by the IRB of General Surgery Department at Ain Shams Faculty of Medicine.
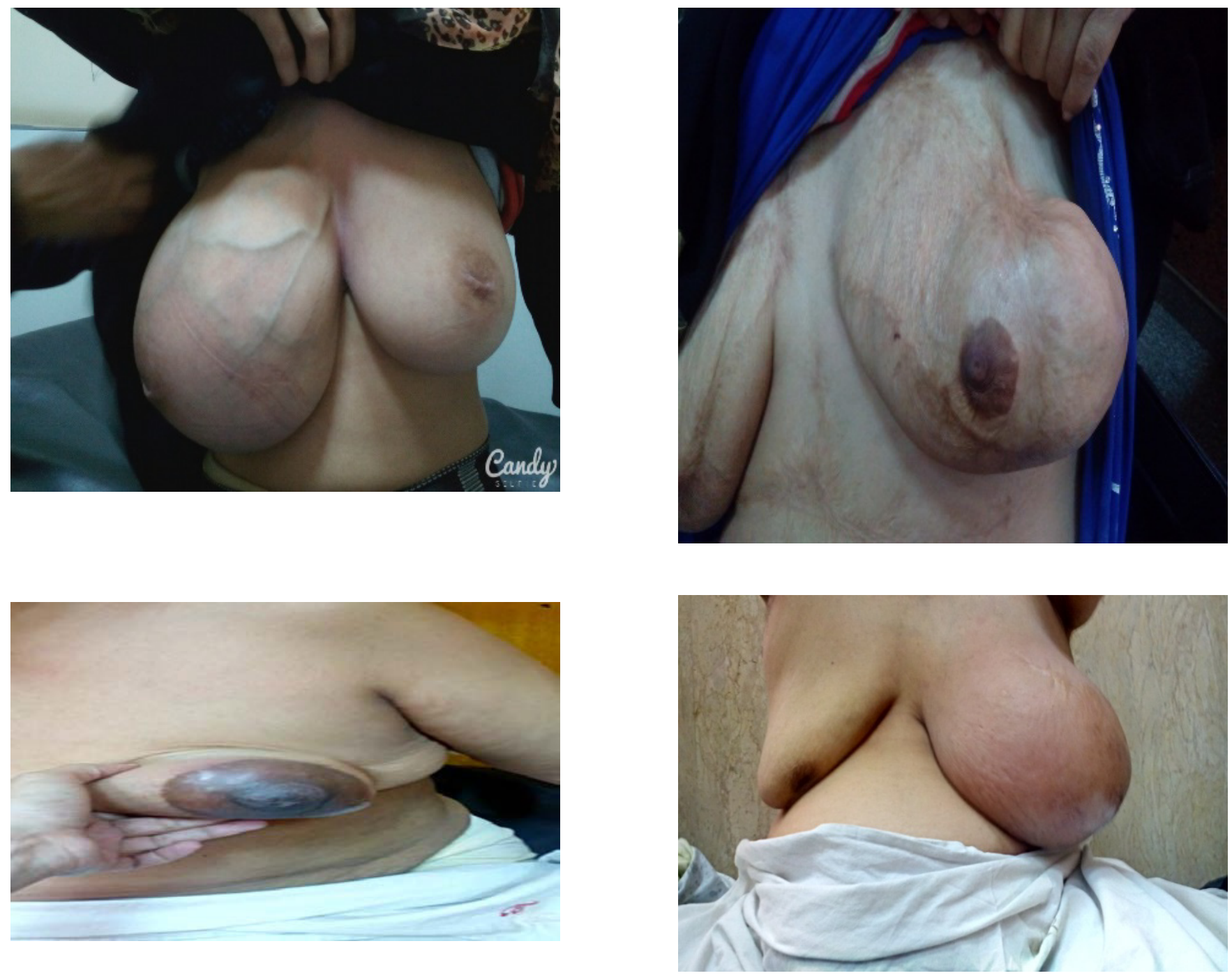

Fig 1: A variety of PT presentations. 

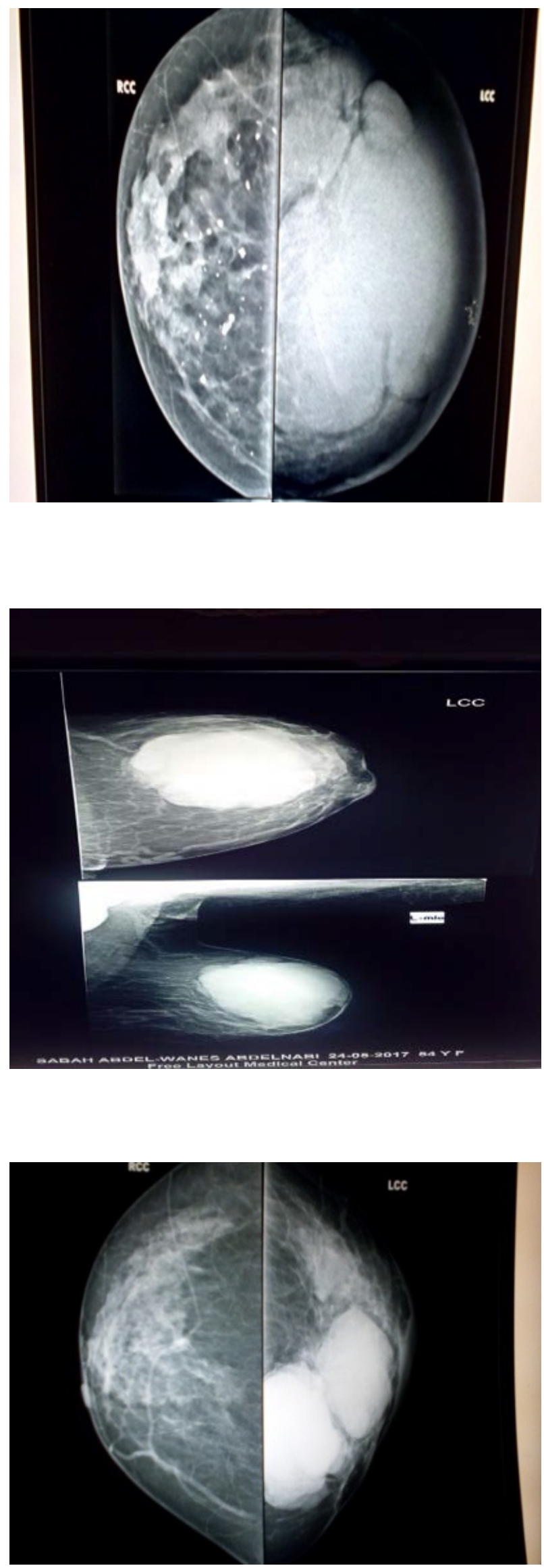

Fig 2: Different mammographic appearances.

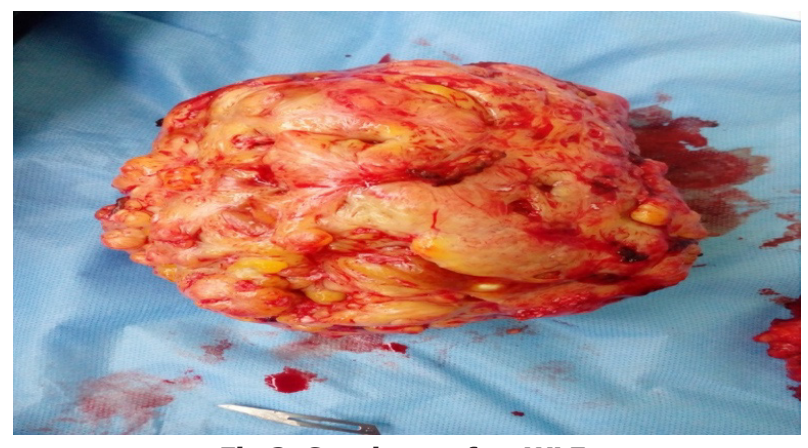

Fig 3: Specimen after WLE.

\section{Statistical analysis of data:}

Statistical presentation and analysis of the present study was conducted using the mean, standard deviation, student t- test, Chi-square tests by SPSS V17. Unpaired Student T-test was used to compare between two groups in quantitative data. P-value was considered significant when it was $\leq 0.05$.

\section{Results}

This study included 24 patients who presented to ASUH along four years starting from August 2014, and were diagnosed to have Phyllodes Tumour (PT). These patients were followed up for a mean of 30 months \pm 10 (range between 20-40 months). Twenty patients $(83.33 \%)$ presented with accidental discovery of a breast mass, whereas only 4 patients $(16.67 \%)$ had pain at the time of assessment. Among our study population, 14 patients $(58.33 \%)$ were recognized to have no associated co-morbidities, left-sided lesions, and benign post-operative histopathological results. Only 4 cases $(16.67 \%)$ showed positive family history. Mastectomy was done in six patients $(50.00 \%)$, while WLE and OS were done in 8 cases (33.335) and in 4 cases $(16.67 \%)$ respectively (Table 1). The mean age of our series was $43.333 \pm 15.808$ (range 21 - 74). Tumour size ranged between 3-15 cm (mean \pm SD 10.083 \pm 4.231 ), while the safety margin in the histopathological reports exceeded $10 \mathrm{~mm}$ in 20 cases $(83.33 \%), 3 \mathrm{~mm}$ and $1 \mathrm{~mm}$ in 2 cases and involved in 2 cases (Table $\mathbf{2}$ ). 
Table 1: Demographic characteristics of study population

\begin{tabular}{|c|c|c|c|}
\hline & & $\mathbf{N}$ & $\%$ \\
\hline \multirow{2}{*}{ Recurrence } & Non-Recurrence & 18 & 75.00 \\
\hline & Recurrence & 6 & 25.00 \\
\hline \multirow{2}{*}{ Co-morbidities } & No Co-morbidities & 14 & 58.33 \\
\hline & Co-morbidities & 10 & 41.67 \\
\hline \multirow{2}{*}{ FH } & Negative & 20 & 83.33 \\
\hline & Positive & 4 & 16.67 \\
\hline \multirow{2}{*}{ Site } & Right & 10 & 41.67 \\
\hline & Left & 14 & 58.33 \\
\hline \multirow{2}{*}{ Presenting symptoms } & Lump & 20 & 83.33 \\
\hline & Pain & 4 & 16.67 \\
\hline \multirow{2}{*}{ Tru cut biopsy } & Non-Phylloid & 14 & 58.33 \\
\hline & Phylloid & 10 & 41.67 \\
\hline \multirow{3}{*}{ Type of surgery } & Mastectomy & 12 & 50.00 \\
\hline & Conservative & 8 & 33.33 \\
\hline & Oncoplastic Surgery & 4 & 16.67 \\
\hline \multirow{2}{*}{ Revision } & Re-excsion & 2 & 33.33 \\
\hline & Oncoplastic Surgery & 4 & 66.67 \\
\hline \multirow{2}{*}{ Post op pathology type Grade } & Benign & 14 & 58.33 \\
\hline & Malignant & 10 & 41.67 \\
\hline \multirow{2}{*}{ Radiotherapy } & Benign & 0 & 0 \\
\hline & Malignant & 4 & 22.09 \\
\hline \multirow{2}{*}{ Safety margin } & Negative & 22 & 91.6 \\
\hline & Positive & 2 & 8.33 \\
\hline
\end{tabular}

Table 2: Demographic characteristics of study population, continued

\begin{tabular}{lcc}
\hline & Range & Mean \pm SD \\
\hline Age (Years) & $21-74$ & $43.333 \pm 15.808$ \\
Size of the tumor (cm) & $3-15$ & $10.083 \pm 4.231$ \\
Disease free period (Years) & $1-1.5$ & $1.250 \pm 0.354$ \\
\hline
\end{tabular}

Six patients (25\%) demonstrated local recurrence after the surgical intervention by a mean of $1.250 \pm 0.354$ (range $1-1.5$ years). Only one case of mortality was recorded during the follow up period. It is worth mentioning that this patient showed several times of recurrence after mastectomy, and then after Transversus Rectus Abdominis Myocutaneous Flap (TRAM), and then after LDF. During the last episode, she experienced life-threatening bleeding from the fungating tumour necessitated blood transfusion, haemostatic dose radiotherapy and angio-embolization.

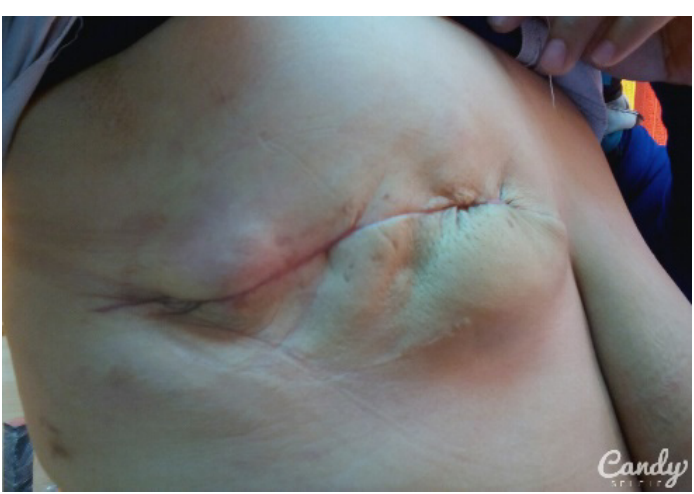

A) LR after MRM. 


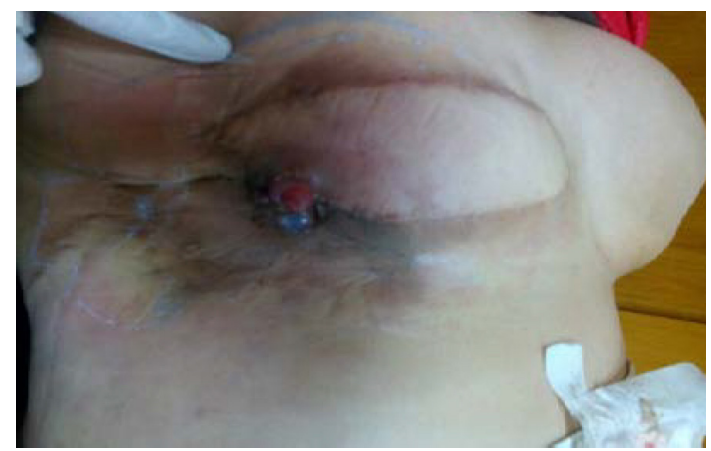

B) LR after LDF.

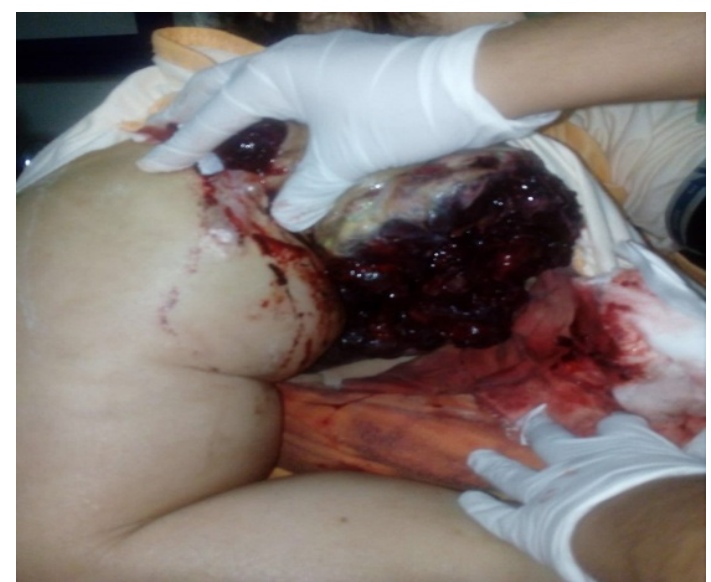

C)

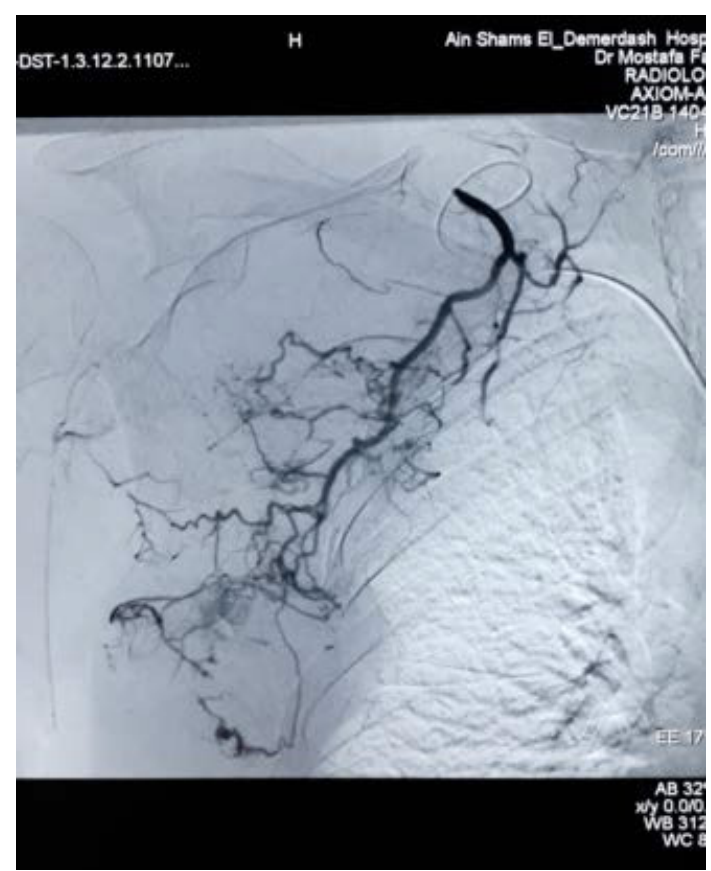

D) Angio-embolization to control bleeding.

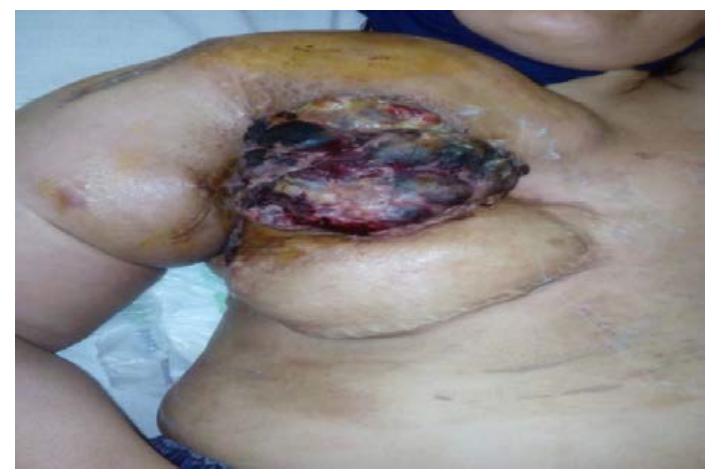

E) After control by angio-embolization.

Fig 4: Aggressive local recurrence after surgical treatment of PT.

The disease-free period for the recurrent cases ranged between $1-1.5$ years (mean \pm SD 1.250 $\pm 0.354)$. Among the recurrent cases, 2 cases (33.33\%) were managed by r-excision and four cases $(66.67 \%)$ by OS in the form of Round Block technique and Latissimus Dorsi Flap (LDF) (Figures 5-7).
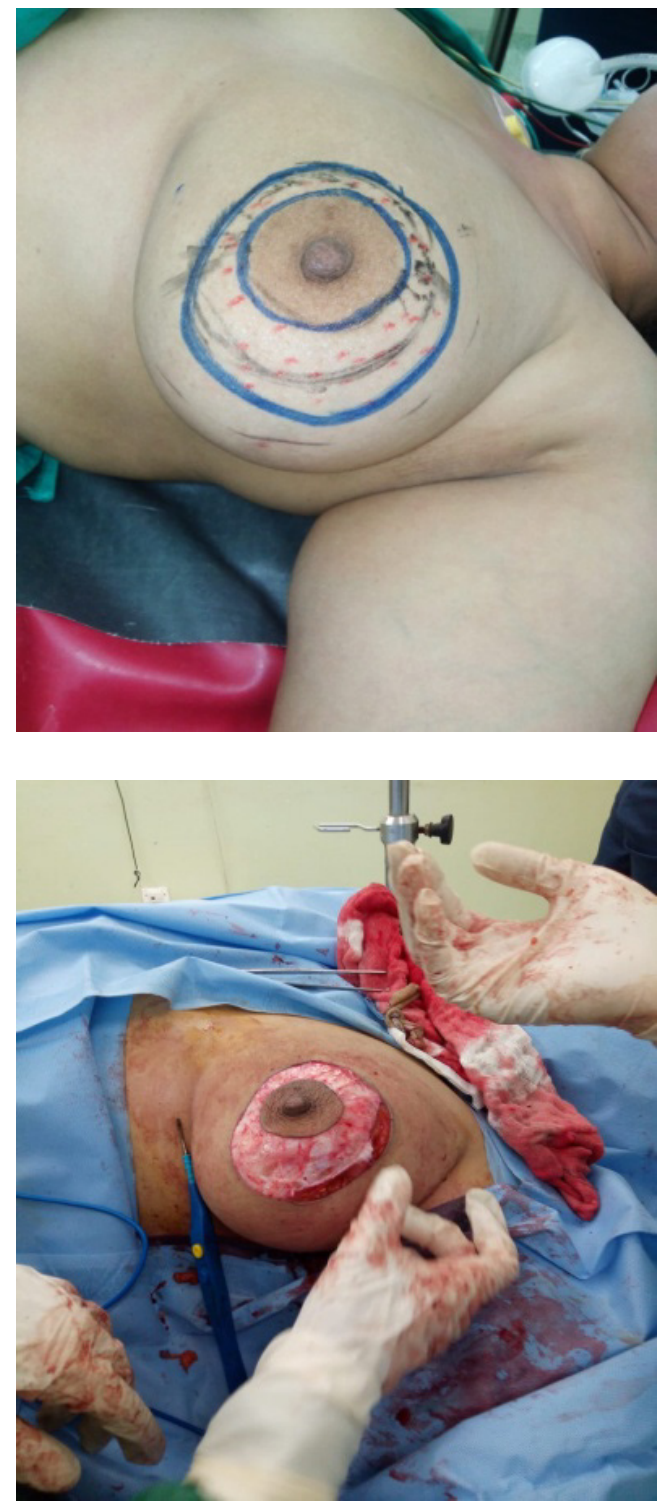


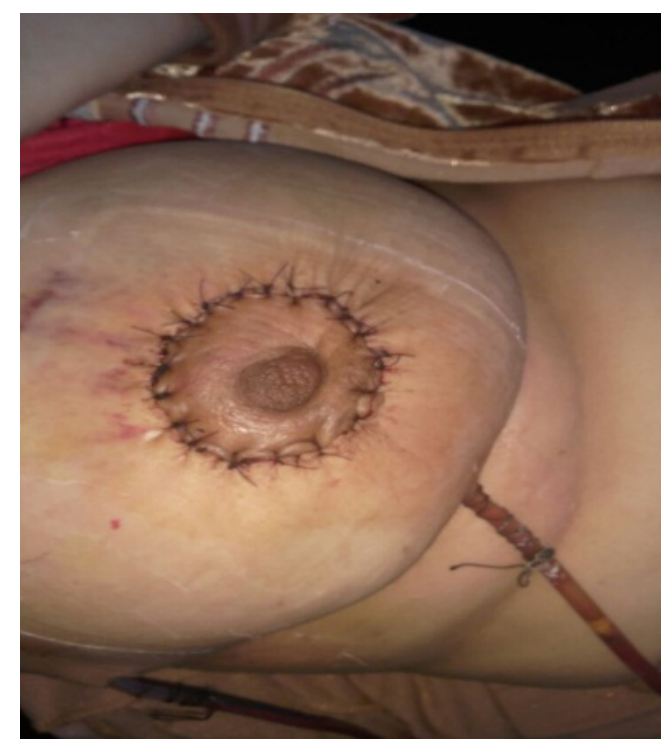

Fig 5: Round Block technique.
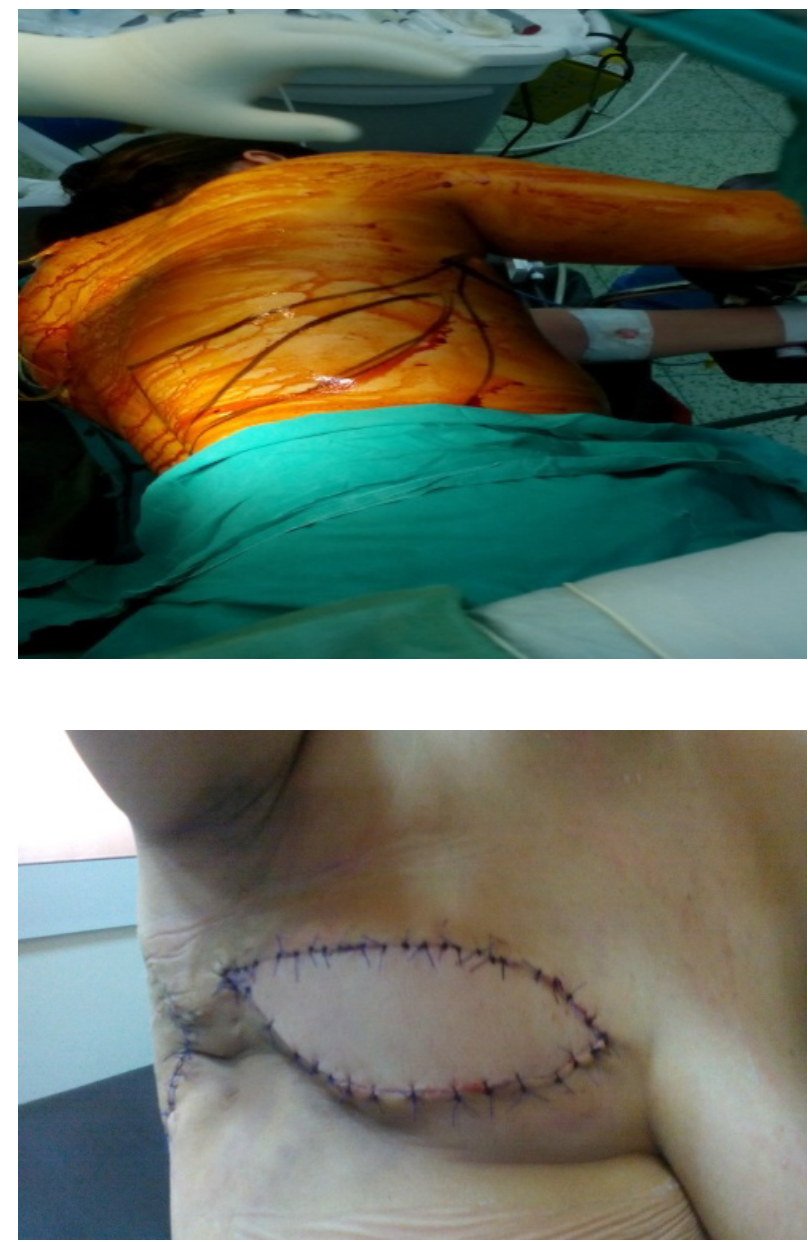

Fig 6: Latissimus Dorsi Flap (LDF) technique.

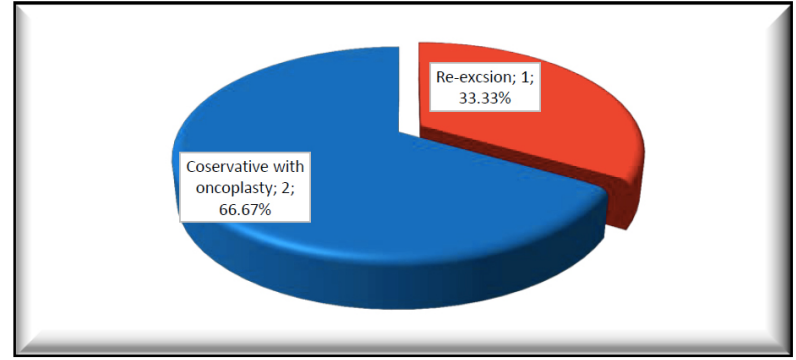

Fig 7: Management of LR following treatment of the primary lesion.

Only 2 recurrent cases (33.33\%) had hypertension, while 4 recurrent patients $(66.67 \%)$ did not have co-morbidities (P-value 0.735). Only 4 cases $(16.67 \%)$ showed positive family history for breast cancer, half of them had local recurrence (P-value 0.371). PTs were located in the left side in 14 patients $(58.33 \%)$, and in the right side in the remaining 10 patients (41.67\%), P-value 0.735 . As a part of triple assessment, tru-cut biopsy showed fibroepthelial lesion highly suggestive of phylloid in 10 patients with clinically suspicious masses (41.67\%), while 14 patients $(58.33 \%)$ trucut biopsy was not conclusive, P-value 0.091 . Half of this cohort underwent Mastectomy, whereas 8 patients $(33.33 \%)$ had WLE and the remaining 4 patients $(16.67 \%)$ had OS in the form of Round Block technique, P-value 0.329. Post-operative histo-pathology revealed malignant PT in 10 patients $(41.67 \%)$ and benign in 14 patients $(58.33 \%)$, P-value 0.735 . Recurrence occurred in the benign PT in 4 cases (66.67\%). Inversely, 8 malignant PTs (44.44\%) did not show any recurrence (Table 3 ). 4 patients with malignant pathology received RT based on MDT recommendations The mean age of the study population was 44.667 (range 21-74) in the non-recurrent arm, and 39.333 (range 2751 ) in the recurrent arm with P-value of 0.636 . The longest diameter of the mass was ranging between 5-15 cm (mean 11.667) and 3-8 cm (mean 5.333) in the non-recurrent group and the recurrent group respectively, P-value 0.016 (Figure 7). (Table 4). 
Table 3: Comparison between recurrent and non-recurrent groups regarding various factors

\begin{tabular}{|c|c|c|c|c|c|c|c|c|c|}
\hline & & \multicolumn{6}{|c|}{ Recurrence } & \multirow{2}{*}{\multicolumn{2}{|c|}{ Chi-Square }} \\
\hline & & \multicolumn{2}{|c|}{ Non-Recurrence } & \multicolumn{2}{|c|}{ Recurrence } & \multicolumn{2}{|c|}{ Total } & & \\
\hline & & $\mathbf{N}$ & $\%$ & $\mathbf{N}$ & $\%$ & $\mathbf{N}$ & $\%$ & $\mathbf{X}^{2}$ & P-value \\
\hline \multirow{2}{*}{ Co-morbidities } & No Co-morbidities & 10 & 55.56 & 4 & 66.67 & 14 & 58.33 & \multirow{2}{*}{0.114} & \multirow{2}{*}{0.735} \\
\hline & Co-morbidities & 8 & 44.44 & 2 & 33.33 & 10 & 41.67 & & \\
\hline \multirow{2}{*}{ FH } & Negative & 16 & 88.89 & 4 & 66.67 & 20 & 83.33 & \multirow{2}{*}{0.800} & \multirow{2}{*}{0.371} \\
\hline & Positive & 2 & 11.11 & 2 & 33.33 & 4 & 16.67 & & \\
\hline \multirow{2}{*}{ Site } & Right & 8 & 44.44 & 2 & 33.33 & 10 & 41.67 & \multirow{2}{*}{0.114} & \multirow{2}{*}{0.735} \\
\hline & Left & 10 & 55.56 & 4 & 66.67 & 14 & 58.33 & & \\
\hline \multirow{3}{*}{ Type of surgery } & Mastectomy & 10 & 55.56 & 2 & 33.33 & 12 & 50.00 & \multirow{3}{*}{2.222} & \multirow{3}{*}{0.329} \\
\hline & BCS & 4 & 22.22 & 4 & 66.67 & 8 & 33.33 & & \\
\hline & Round block technique & 4 & 22.22 & 0 & 0.00 & 4 & 16.67 & & \\
\hline \multirow{2}{*}{ Pos-op pathology Nature } & Benign & 10 & 55.56 & 4 & 66.67 & 14 & 58.33 & \multirow{2}{*}{0.114} & \multirow{2}{*}{0.735} \\
\hline & Malignant & 8 & 44.4 & 2 & 33.33 & 10 & 41.67 & & \\
\hline
\end{tabular}

The mean age of the study population was 44.667 (range 21-74) in the non-recurrent arm, and 39.333 (range 27-51) in the recurrent arm with P-value of 0.636 . The longest diameter of the mass was ranging between $5-15 \mathrm{~cm}$ (mean 11.667) and 3-8 $\mathrm{cm}$ (mean 5.333) in the non-recurrent group and the recurrent group respectively, P-value 0.016 (Figure 7). (Table 4).

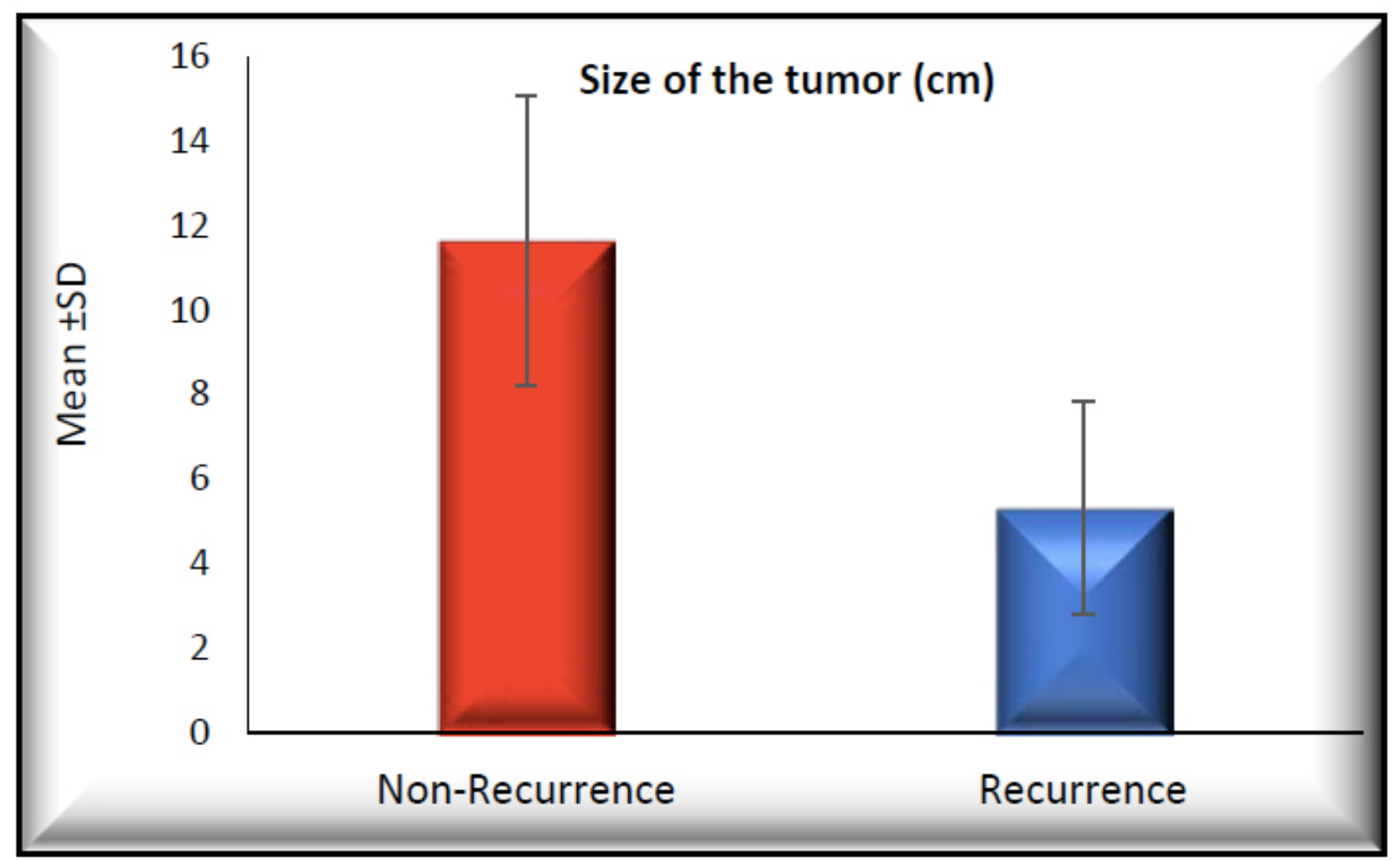

Fig 8: Relation between tumour size and its local recurrence. 
Table 4: Impact of age, tumor size and safety margin on LR

\begin{tabular}{lccccc}
\hline & & \multicolumn{2}{c}{ Recurrence } & \multicolumn{2}{c}{ T-Test } \\
\cline { 3 - 6 } & & Non-Recurrence & Recurrence & t & P-value \\
\hline \multirow{2}{*}{ Age (Years) } & Range & $21-74$ & $27-51$ & 0.488 & 0.636 \\
& Mean $\pm S D$ & $44.667 \pm 17.306$ & $39.333 \pm 12.014$ & & \\
Size of the tumor $(\mathbf{c m})$ & Range & $5-15$ & $3-8$ & 2.909 & $0.016 *$ \\
& Mean $\pm S D$ & $11.667 \pm 3.428$ & $5.333 \pm 2.517$ & & \\
\hline
\end{tabular}

\section{Discussion}

Demographic characteristics, such as co-morbidities, family history, tumor site, type of surgery, tumor grade, age and safety margin, failed to prove statistical significance on the incidence of the LR after surgical management for PTs. However, tumor size alone was found to be independent risk factor for LR.

Our study indicates that LR can occur after surgical excision of benign PTs. There are reports of progression of benign PTs to more aggressive subtypes if they recur, this is extremely rare and its importance is possibly overestimated.? This typically took place with one of the recurrent cases in this series. Her initial tru-cut pathology revealed benign PT. But within few months, PT recurred in a wild behavior for several times mandating repeated surgical revisions, adjuvant radiotherapy and even angio-embolization to control the cruel local disease. The biological behavior and pathological features of phyllodes tumors are variable, as benign phyllodes tumors may be associated with recurrence rate of $10-40 \%$. meanwhile, malignant phyllodes tumors may show good clinical course. ${ }^{8}$

In an analysis of 70 patients with malignant phyllodes tumors, Mitus et al. observed patients who underwent mastectomy (82.4\%) versus wide local excision $(83.3 \%)$ with no evidence of recurrence after 5 years. ${ }^{9}$ In our series, LR occurred after 1-1.5 years. This might propose the Management of the large phyllodes tumors presents the surgeon with unique challenges. Complete excision, with accurate histological examination and continued follow-up care, is the best way to treat phyllodes tumors. In most cases wide local excision is indicated, with an adequate margin. Although no absolute consensus regarding margin size have been established a 1 $\mathrm{cm}$ macroscopic at the time of the operation and $1 \mathrm{~mm}$ microscopic margin for most cases has been advocated as adequate. ${ }^{10}$ Most authors consider excision margin to be positive if the tumor was present at or close to $(<1 \mathrm{~mm})$ the inked tissue on histo-pathology. In fact, taking into account that local recurrence of phyllodes is high and that an affected margin is the single independent predictor of local recurrence is therefore understandable the paramount significance of the wide excision in the local recurrence-free rate. ${ }^{11}$

The value of negative margins was demonstrated in several studies, due to higher correlation with disease recurrence. ${ }^{12-14}$ In one of these studies, Spitaleri et al. reported that mean local recurrence rate reached $31.5 \%$ in patients with positive surgical margins. ${ }^{12}$ Furthermore, Lim et al. identified margin status as the only parameter affecting overall survival at 5 years. ${ }^{13}$ Although the NCCN recommends a clearance of at least $1 \mathrm{~cm}$, a recent literature review by Shaaban et al. found no statistically significant difference in recurrence regardless of $1 \mathrm{~mm}$ versus $10 \mathrm{~mm}$ margins. ${ }^{14}$ The latter study's results are consistent with our results which did not prove any significant difference between margins below $1 \mathrm{~cm}$ and those higher than $1 \mathrm{~cm}$. However, the authors noted that the possibility of recurrence based on margins status potentially differs depending on tumor behavior, and those with benign PTs do not mandate excision of $>1 \mathrm{~cm}$. however, more research is needed in this area.

In the literature the average size of phyllodes tumors is around $4-8 \mathrm{~cm}$ varying from 1 to over $40 \mathrm{~cm}$ at the extremes. ${ }^{15}$ In the study by Eugenie Guillot and his co-workers, the average size was 3 $\mathrm{cm}$ with a range of $0.5-15 \mathrm{~cm}$, the largest tumors were of the highest grade. In the same series, univariate analysis showed tumor size to be a significant prognostic indicator for local control $(p=0.02)$, which is consistent with other authors. ${ }^{16}$ In our study, smaller PTs paradoxically demonstrate higher malignant potential than the larger ones, which is contradicting the classical conception of the proportional relationship between tumor size and its aggressiveness. Moreover, large sized masses were not associated with higher rate of recurrence mostly due to aggressive initial excision by our surgeon. This was consistent with evidence from literature that small Phylloid may be undertreated by simple enucleation may have higher potential of LR.

BCS with adequate margins $(\geq 1 \mathrm{~cm})$ is the mainstay primary therapy for non-metastatic PT. ${ }^{17}$ In the same review, it is worth to mention the fact that local recurrence rate between conservation 
and mastectomy were not statistically significant provided the margins of excision were adequate. ${ }^{11}$ This was consistent with our results which revealed no statistical impact of mastectomy versus WLE on LR rates. Axillary dissection was not performed in our series, except for axillary sampling of one case with axillary lymphadenopathy, since nodal metastases secondary to PT are very rare. Recent studies advocate the use of radiotherapy for borderline and malignant PT, recurrent tumors and in cases where it is not possible to achieve a greater than one-centimeter surgical margin. ${ }^{18}$ Given the rarity of PT, it might be challenging to derive a randomized controlled trial with adequate sample size.

Although limited in the literature, reports of immediate breast reconstruction have been produced. Immediate breast reconstruction with implant only autologous tissue alone or in combination with an implant did not warrant a higher recurrence rate compared to simple mastectomy and also it did not interfere with follow up or the detection of recurrent lesions. In the appropriate setting nipple sparing skin-sparing mastectomy can be applied without affecting recurrence rates something that is in the author's previous personal experience. The same applies for breast conservation Oncoplastic approach in the treatment of wide excisions and local recurrence being no different provided adequate margins are achieved. ${ }^{11}$ In our series, only two patients underwent OS in the form of Round Block technique. In the recurrent patients, tumours were managed by Round Block technique and Latissimus Dorsi Flap (LDF).

In addition to the prognostic factors of PTs, several recent studies examining genetic characteristics of PTs have found correlations between varying genomic alterations and PTs. ${ }^{19,20}$ These alterations could potentially add apiece of knowledge regarding therapeutic targets and better management of this rare disease. Genetic analysis will potentially support histological evaluation for better understanding of the recurrence nature of this rare tumor.

\section{Limitations of the study}

There are several limitations in the present study. Due to low numbers and paucity of the disease, the significance of some parameters may not have been identified in under-powered analyses. This study represents a single institute experience of a relatively rare disease with the subsequent small sample size. Incidence of PTs could not be calculated because ASUH is a referral hospital from all over Egypt. Eventually, pathological details regarding prognostic factors such as stromal overgrowth, mitotic index and cellular atypia) were not available in the analysis.

Therefore, we recommend adoption of future multi-centeric and prospective studies including full pathological details and highlighting both LR and distant metastases.

\section{Conclusion}

Benign and small PTs should be excised with adequate safety margin rather than simple enucleation, and their malignant potential and LR liability should be taken into consideration. However, multi-centric studies with large population are considered a golden key in understanding the recurrence behaviour of this rarely-occurring disease.

\section{Conflict of Interest}

None to declare

\section{Financial Support}

None to declare

\section{References}

1. Farias-Eisner GT, Small K, Swistel A, Ozerdem $\mathrm{U}$, Talmor M: Immediate implant breast reconstruction with acellular dermal matrix for treatment of a large recurrent malignant phyllodes tumor. Aesthetic Plast Surg. 2014; 38(2): 373-8. doi: 10.1007/s00266-014-02839. PMID: 24570179.

2. Lee AHS: Recent developments in the histological diagnosis of spindle cell carcinoma, fibromatosis and phyllodes tumour of the breast: Spindle cell and fibroepithelial lesions. Histopathology. 2007; 52(1): 45-57.

3. Norris HJ, Taylor HB: Relationship of histologic features to behavior of cystosarcoma phyllodes. Analysis of ninety-four cases. Cancer. 1967; 20: 2090-9.

4. Parker SJ, Harries SA: Phyllodes tumours. Postgrad Med J. 2001; 77(909): 428-435.

5. G. Cohn-Cedermark, L. E. Rutqvist, I: Rosendahl, C. Silfversward: Prognostic factors in cystosarcoma phyllodes: A clinicopathologic study of 77 patients," Cancer. 1991; 68(9): 2017-2022.

6. G. Singh, RK Sharma: Immediate breast reconstruction for phyllodes tumors, Breast. 2008;17(3):296-301.

7. Jang JH, Choi MY, Lee SK, et al: Clinicopathologic risk factors for the local recurrence of phyllodes tumors of the breast. Ann Surg Oncol. 2012; 19(8): 2612-2617.

8. Jie Ren, Liyan Jin, Bingjing Leng et al: Surgical excision and oncoplastic breast surgery in 32 
patients with benign phyllodes tumors. World Journal of Surgical Oncology. 2018; 16: 153.

9. Mitus J, Reinfuss M, Mitus JW, et al: Malignant phyllodes tumor of the breast: Treatment and prognosis. Breast J. 2014; 20(6): 639e44.

10. Pitsinis V, Moussa O, Hogg F, McCaskill J: Reconstructive and oncoplastic surgery for giant phyllodes tumors: A single center's experience. World J Plast Surg. 2017; 6(2): 233-237.

11. McKelvey MT, Gordillo G, Nuovo GJ, Carson WE $3^{\text {rd }}$ : Giant breast tumors: Surgical management of phyllodes tumors, potential for reconstructive surgery and a review of literature. World $\boldsymbol{J}$ Surg Oncol. 2008; 6: 117.

12. Spitaleri G, Toesca A, Botteri E, Bottiglieri L, Rotmensz N, Boselli S, et al: Breast phyllodes tumor: A review of literature and a single center retrospective series analysis. Crit Rev Oncol Hematol. 2013; 88(2): 427-436.

13. Lim SZ: Breastsarcomas and malignant phyllodes tumours: Comparison of clinicopathological features, treatment strategies, prognostic tumours: A comparison of clinicopathological features, treatment strategies, prognostic factors and outcomes. Breast Cancer Res Treat. 2016; 159(2): 229-244.

14. Shaaban M, Barthelmes L: Benign phyllodes tumours of the breast: (Over) treatment of margins - a literature review. Eur J Surg Oncol. 2017; 43: 1186-1190.

15. Bouhafa T, Masbah O, Bekkouch I, et al: Phyllodes tumors of the breast: Analysis of 53 patients. Cancer radiother. 2009: 85-91. 26. Belkacemi $Y$, Bousquet $G$, Marsiglia $H$, et al. Phyllodes tumor of the breast. Int $\boldsymbol{J}$ Radiat Oncol Biol Phys. 2008; 70: 492-500.

16. Guillot E, Couturaud B, Reyal F, Curnier A, Ravinet J, Lae M, et al: Management of phyllodes breast tumors. The Breast Journal. 2011; 17(2): 129-137.

17. A.W. Chaney, et al: Primary treatment of cystosarcoma phyllodes of thebreast, Cancer. 2000; 9(7): 1502-1511.

18. C.A. Ossa, et al: Phyllodes tumor of the breast: a clinic-pathologic study of 77cases in a Hispanic cohort, Colomb. Med. (Cali.). 2015; 6(3): 104108.

19. Nozad S, Sheehan CE, Gay LM, et al: Comprehensive genomic profiling of malignant phyllodes tumors of the breast. Breast Cancer Res Treat. 2017; 162(3): 597-602.

20. Kim JY, Yu JH, Nam SJ, et al: Genetic and clinical characteristics of phyllodes tumors of the breast. Transl Oncol. 2017; 11(1): 18-23. 